\title{
Pressure-induced phase transformation and structural resilience of single-wall carbon nanotube bundles
}

\author{
Surinder M. Sharma, ${ }^{1}$ S. Karmakar, ${ }^{1}$ S. K. Sikka, ${ }^{1}$ Pallavi V. Teredesai, ${ }^{2}$ A. K. Sood ${ }^{2,3}$ A. Govindaraj, ${ }^{3}$ and C. N. R. Rao ${ }^{3}$ \\ ${ }^{1}$ High Pressure Physics Division, Bhabha Atomic Research Center, Mumbai 400 085, India \\ ${ }^{2}$ Department of Physics, Indian Institute of Science, Bangalore 560 012, India \\ ${ }^{3}$ Chemistry and Physics of Materials Unit, Jawaharlal Nehru Centre for Advanced Scientific Research, Jakkur Campus, \\ Jakkur P.O., Bangalore 560 064, India \\ (Received 12 January 2001; published 2 May 2001)
}

\begin{abstract}
We report here an in situ x-ray diffraction investigation of the structural changes in carbon single-wall nanotube bundles under quasihydrostatic pressures up to $13 \mathrm{GPa}$. In contrast with a recent study [Phys. Rev. Lett. 85, 1887 (2000)] our results show that the triangular lattice of the carbon nanotube bundles continues to persist up to $\sim 10 \mathrm{GPa}$. The lattice is seen to relax just before the phase transformation that is observed at $\sim 10$ GPa. Further, our results display the reversibility of the two-dimensional lattice symmetry even after compression up to $13 \mathrm{GPa}$ well beyond the $5 \mathrm{GPa}$ value observed recently. These experimental results explicitly validate the predicted remarkable mechanical resilience of the nanotubes.
\end{abstract}

DOI: 10.1103/PhysRevB.63.205417

PACS number(s): $61.48 .+\mathrm{c}, 61.10 . \mathrm{Nz}, 61.50 . \mathrm{Ks}, 81.40 . \mathrm{Vw}$

\section{INTRODUCTION}

Due to the quasi-one-dimensional structure, single-wall carbon nanotubes (SWNTs) have been shown to have some unique and interesting physical properties. ${ }^{1}$ These nanotubes have also been predicted to have extraordinary mechanical properties such as enormous flexibility in terms of complete structural reversibility on bending up to $110^{\circ} .^{2}$ In addition, molecular dynamics (MD) simulations predict that SWNTs may undergo fully reversible morphological changes under extreme deformations. ${ }^{3,4}$ The synthesis of bundles of singlewall carbon nanotubes, with a narrow size distribution, has provided tremendous impetus to the experimental investigations. ${ }^{5}$ Several high-pressure Raman investigations have been carried out recently. ${ }^{6-9}$ In all of these studies, Raman intensity reduces dramatically beyond a few GPa and this has been suggested to be due to the loss of the electronic resonance in the Raman scattering cross-section because of the faceting of the neighboring tubes. ${ }^{6}$ A slight change in the slope of pressure-induced Raman shifts at $\sim 1.7 \mathrm{GPa}$ has also been ascribed to a structural transformation from a triangular to a monoclinic lattice. ${ }^{7}$ Recent Raman investigations by Teredesai et al. $^{9}$ indicate a structural phase transition near 10 $\mathrm{GPa}$. This was conjectured to be due to faceting, as the frequency of the tangential mode approaches that of graphite. Also, all the Raman studies indicate reversibility of behavior upon unloading of the pressure. In particular, the data of Ref. 9 demonstrated the reversibility on pressure release from 25.9 GPa. However, due to the lack of information about the structural evolution of SWNTs under pressure, it was not possible to unambiguously relate these measurements to the microscopic changes in the SWNTs. A recent X-ray diffraction study ${ }^{10}$ of SWNTs under pressure, suggests the vanishing of the triangular lattice at $\sim 1.5 \mathrm{GPa}$ and its regeneration if unloaded from less than $4 \mathrm{GPa}$. Beyond $5 \mathrm{GPa}$, these $\mathrm{x}$-ray results indicate an irreversible change in total contrast to the results of Ref. 9. High-pressure behavior of SWNTs has also been investigated under nonhydrostatic stresses, using a piston-cylinder device without any pressure medium. This study, restricted to $\sim 2.9 \mathrm{GPa}$, displays a reversible increase in the density of SWNTs to almost that of graphite. ${ }^{11}$ This has been speculated to be due to the crushing or flattening of the cross section of the nanotubes from circular to elliptical shape under the nonhydrostatic stresses. So, to find a consistent interpretation of several experimental results mentioned above, it is necessary to investigate SWNTs using an intense $\mathrm{X}$-ray radiation as the diffracted intensities are likely to be rather weak. In addition, it will be interesting to compare the behavior of SWNTs with that of other carbon polymorphs, e.g., $\mathrm{C}_{60}$ and $\mathrm{C}_{70}$ fullerenes that undergo irreversible and reversible pressure-induced amorphization. ${ }^{12-14} \mathrm{We}$ present here an in situ x-ray diffraction investigation of SWNT bundles and relate the results to other experimental and theoretical studies.

\section{EXPERIMENTAL DETAILS}

SWNT bundles were prepared by the standard arc discharge method. For this, a composite rod, made by filling powders of graphite, $\mathrm{Y}_{2} \mathrm{O}_{3}(1$ at. $\% \mathrm{Y})$ and $\mathrm{Ni}(4.2$ at. $\% \mathrm{Ni})$ in a hole, was used as an anode and a simple graphite rod as a cathode. The material produced through a dc arc in $\mathrm{He}$ atmosphere was appropriately washed with several chemicals, decanted, filtered, dried, and characterized by transmission electron microscopy. ${ }^{9,15}$ At ambient conditions, the $\mathrm{x}$-ray diffraction peak corresponding to $(1,0)$ plane of the two-dimensional triangular lattice shows that our SWNTs correspond to a lattice constant $\mathbf{a}_{0}$ of $17.97 \AA$. As the curvature of the nanotubes reduces the contact area on which the repulsive forces act on the tubes, the intertube gap is expected to be smaller than the (002) spacing of graphite. If the intertube gap is taken as $3.12 \AA$, ${ }^{10}$ present SWNTs correspond to a tube diameter of $14.85 \AA$. Therefore, our sample consists of either $(11,11)$ armchair tubes or $(19,0)$ zigzag tubes or any other appropriate combination of integers $n$ and $m{ }^{17}$ Thermogravimetric analysis of the sample showed graphite abundance to be $\sim 8 \%$. The sample, containing ran- 
domly oriented rope bundles of $\sim 100 \AA$ diameter and several micron length, was loaded in a steel gasket hole ( $\sim 200 \mu \mathrm{m}$ diameter) of a diamond anvil cell (DAC). A tiny ruby chip was used to measure the pressure with methanolethanol (4:1) as a pressure transmitter. Angle dispersive X-ray diffraction experiments on SWNTs were carried out up to a pressure of $13 \mathrm{GPa}$ at the beamline BL10XU of SPring8, using a monochromatized $x$-ray beam of $1 \AA$. The diffraction patterns were recorded using the imaging plate kept at a distance of $\sim 25 \mathrm{~cm}$ from the DAC. Two dimensional imaging plate records were transformed to one dimensional diffraction profiles by the radial integration of the diffraction lines.

\section{RESULTS AND DISCUSSION}

The triangular two-dimensional lattice of SWNTs gives the strongest feature at $\sim Q_{100}=0.402 \AA^{-1}$ and on the imaging plate it shows up as a diffraction circle close to the direct spot. No additional line of SWNT could be observed due to the fact that other diffraction peaks are much weaker than the first. ${ }^{5}$ Also, the small volume of the sample in the DAC further reduces the observed intensities. Figure 1 shows variations in the intensity of the diffraction pattern corresponding to the two-dimensional triangular lattice as a function of pressure, on loading, as well as on release of pressure. The most important feature is that on increasing the pressure, the SWNT diffraction line vanishes beyond $\sim 10 \mathrm{GPa}$ [Fig. 1(c)]. On release of pressure, from $\sim 13$ to $\sim 7 \mathrm{GPa}$, the diffracted intensity corresponding to SWNTs reappears [Fig. 1(d)] and eventually regains almost full initial intensity on complete release of the pressure [Fig. 1(e)]. The vanishing intensity of the diffraction profile of SWNTs indicates a phase transformation. However, we do not see any new diffraction lines across this pressure. This implies that either the new structure is such that it does not have any strong Bragg reflections or it has lost the translational coherence in a bundle. Alternatively, if the single-wall nanotubes graphitize at this pressure, then such a change will be irreversible as has been seen earlier. ${ }^{16}$ Also, our calculated diffraction patterns with totally circular and ideal hexagonal tubes show that faceting does not change the diffracted intensity of the first diffraction line significantly. (Moreover, recent MD calculations suggest that SWNTs are faceted even at ambient pressures. ${ }^{610}$ ) Therefore, we feel that the complete loss of the low-angle diffraction line due to the triangular lattice of the SWNT at $10 \mathrm{GPa}$ on compression and its retrieval on decompression clearly demonstrates a reversible loss of translational coherence. These results establish that the structural changes at $\sim 10 \mathrm{GPa}$ are not related to the uniform faceting and/or uniform flattening of the tubes as speculated in some earlier studies. ${ }^{9,11}$ Further, the re-emergence of lattice on release of pressure provides a direct confirmation of the theoretical predictions that most structural changes in the SWNT are totally reversible. ${ }^{2,3}$ These results are distinctly different from those of Tang et al. ${ }^{10}$ on two counts. First the diffraction signal for $(100)$ line of our $(11,11)$ armchair or equivalent tubes vanishes at $\sim 10 \mathrm{GPa}$, in contrast with the observed loss of diffracted intensity at $\sim 1.5 \mathrm{GPa}$ for $(10,10)$
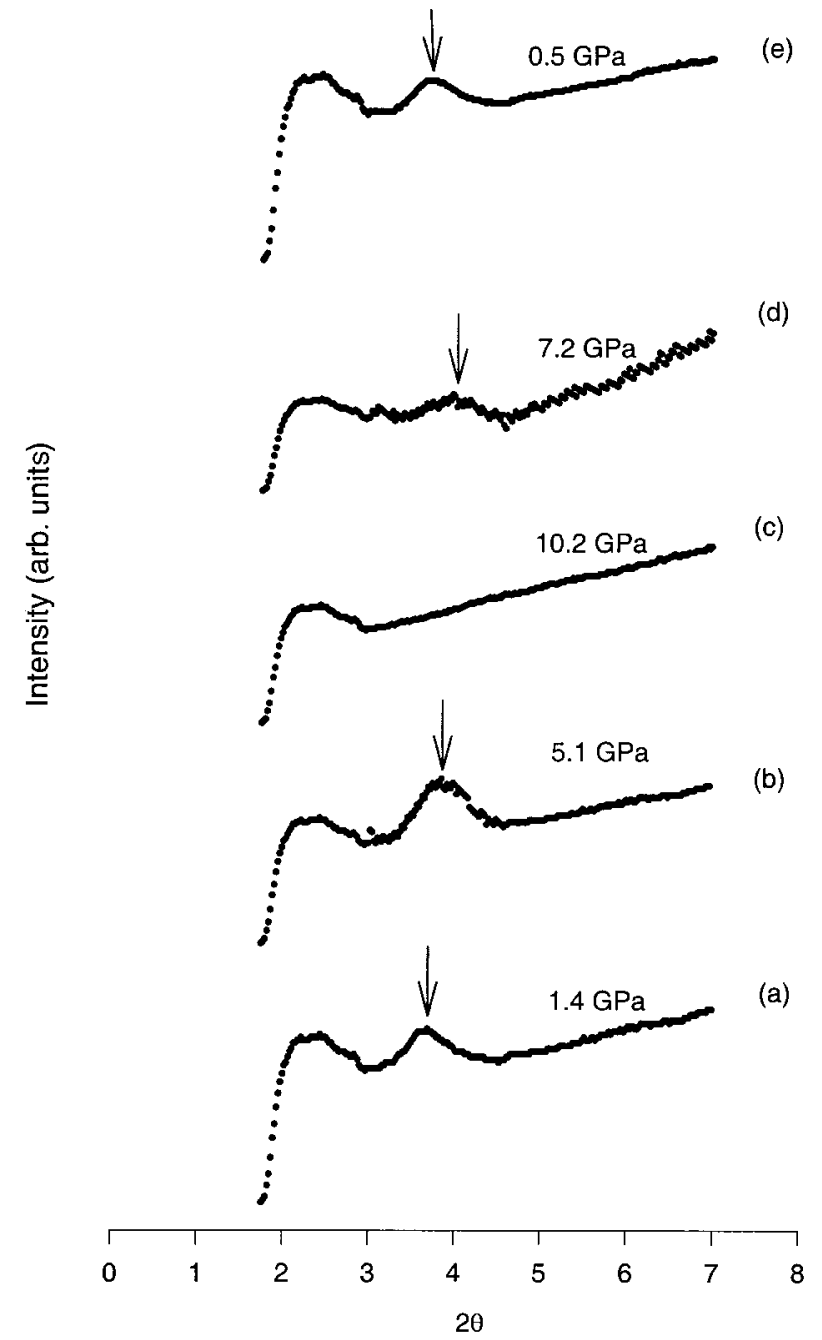

FIG. 1. One-dimensional x-ray diffraction profile of SWNTs from the $(1,0)$ planes of the two-dimensional triangular lattice at various pressures. (a) $1.4 \mathrm{GPa}$, (b) $5.1 \mathrm{GPa}$, (c) $10.2 \mathrm{GPa}$ represents the records on increasing pressure while (d) $7.2 \mathrm{GPa}$ and (e) 0.5 $\mathrm{GPa}$ correspond to the decreasing pressure.

or equivalent tubes of Tang et al. ${ }^{10}$ Second, our results establish the reversibility of transformation from unloading of pressure from $\sim 13 \mathrm{GPa}$, in comparison with the corresponding limit of $4 \mathrm{GPa}$ observed by Tang et al. ${ }^{10} \mathrm{We}$ should also point out that the reversibility observed in our experiments is consistent with the reversible behavior observed in Raman experiments carried on the same batch of samples. ${ }^{9}$ However, as none of the theoretical work carried out so far predicts a strong dependence of the deformational behavior on the tube diameter, it is difficult to rationalize the observed different behavior for $(11,11)$ (or equivalent) and $(10,10)$ (or equivalent) tubes. Future work may provide some understanding of these results.

In Fig. 2, we show the variation of the $d$ spacings of various diffraction lines with pressure as observed in this experiment. Up to $\sim 8 \mathrm{GPa}$, we discern a systematic compression as displayed by the reduction of $d_{100}$ of SWNT as a function of pressure. Within the experimental resolution, the absence of any additional diffraction lines or discernible 


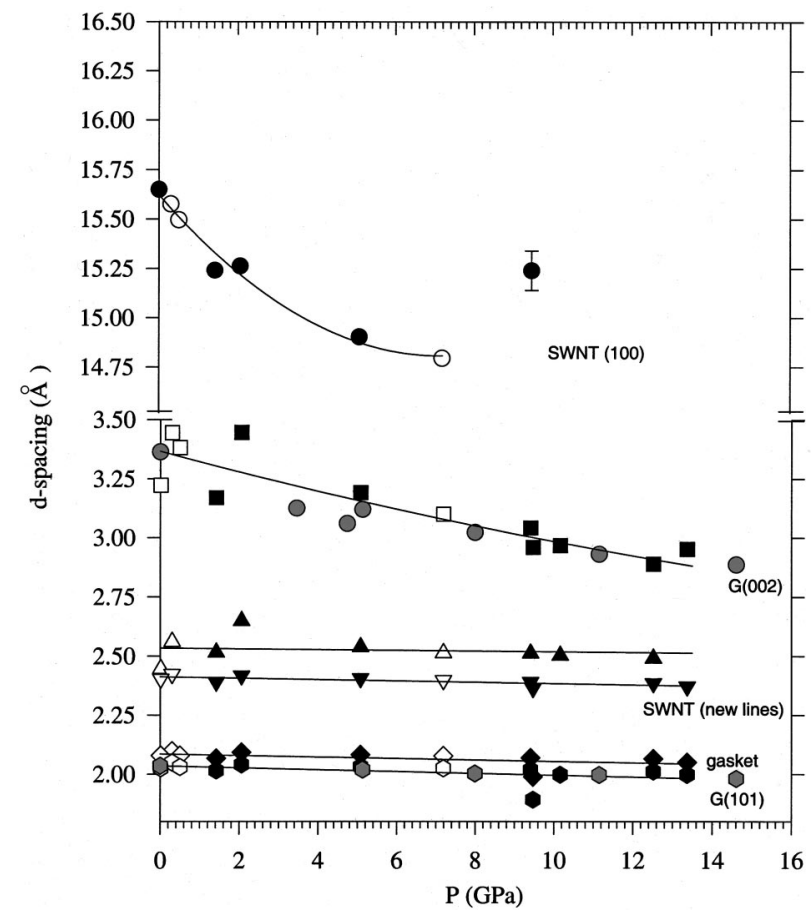

FIG. 2. Pressure variation of the $d_{h k l}$ values. Filled symbols represent the data in an increasing pressure cycle while open symbols correspond to a decreasing pressure cycle. Hatched symbols are the published data of graphite from Ref. 23.

broadening due to the splitting of the diffraction line (owing to proposed lowering of symmetry across $1.7 \mathrm{GPa}$ ) in our data do not support the suggestion of a structural transformation at this pressure. ${ }^{7}$ Observed variations in the lattice spacing also highlights the differences in the behavior of SWNT under hydrostatic and non-hydrostatic stress conditions. For example, by $5 \mathrm{GPa}$, the basal plane lattice constant reduces to $\sim 0.95 a_{0}$. If we assume that the compression along the tube axis to be the same as that in the basal plane of graphite, ${ }^{18}$ these results suggest that at $\sim 5 \mathrm{GPa}, V / V_{0}$ $=0.9$. In contrast, the compression under nonhydrostatic conditions of the piston cylinder apparatus is $V / V_{0}=0.75$ at $\sim 2.5 \mathrm{GPa} .{ }^{11}$ The variation of the two-dimensional triangular lattice parameter a with pressure can be fitted to the onedimensional analogue ${ }^{18}$ of the Murnaghan equation, ${ }^{19} a / a_{0}$ $=\left[\left(\beta^{\prime} / \beta_{0}\right) P+1\right]^{-1 / \beta^{\prime}}$ where $\beta_{0}$ is the bulk modulus and $\beta^{\prime}$ is the pressure derivative. Our data up to $\sim 8 \mathrm{GPa}$, taken from the increasing as well as decreasing pressure runs, can be fitted with $\beta_{0}=43( \pm 4) \mathrm{GPa}$ and $\beta^{\prime}=33( \pm 3)$. Compare this with $c$-axis compression of graphite, which has $\beta_{0}$ $=35.7 \mathrm{GPa}$ and $\beta^{\prime}=10.8 .^{18}$ This shows that up to $\sim 8 \mathrm{GPa}$, SWNTs are somewhat less compressible than graphite along the $c$ axis. Assuming the tube axial compression as that of the graphite basal plane, we find the low-pressure bulk modulus to be $\sim 34 \mathrm{GPa}$.

Several authors have computed the elastic properties of SWNTs. ${ }^{4,20,21}$ Figure 3 compares our observed bulk modulus as well as that of Tang et al. ${ }^{10}$ with the results of these calculations. Our results are in excellent agreement with the theoretical estimates of Tersoff and Ruoff ${ }^{4}$ and Popov, Van

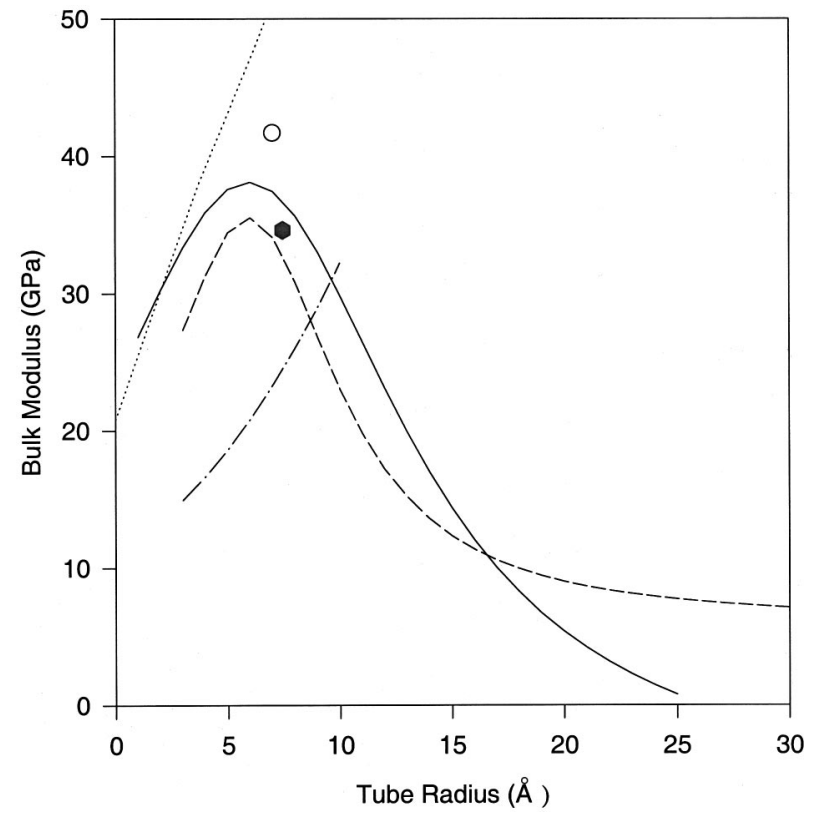

FIG. 3. Comparison between the observed bulk modulus for $(11,11)$ or equivalent tubes (present work, represented by a solid hexagon) and for $(10,10)$ or equivalent tubes (Ref. 10, represented by an open circle) and the theoretical results (Refs. 4,20,21). The dashed line represents the calculated results of Tersoff and Ruoff (Ref. 4), the dash dot corresponds to the model calculations of $\mathrm{Lu}$ (Ref. 21), the dotted line represents the computed results of Popov, Van Doren, and Balakanski (Ref. 20) in which rigid tubes interact through van der Waal forces and the solid line corresponds to their more detailed calculations in which interacting elastically deformable tubes are treated (Ref. 20).

Doren, and Balakanski ${ }^{20}$ [which are, respectively, 34 and 37.3 GPa for $(11,11)$ or equivalent tubes]. Observed bulk modulus of Tang et al., though slightly higher $(\sim 41.7 \mathrm{GPa})$, is also in reasonable agreement with the theoretical calculations. Figure 3 also shows that results of the present investigation and of Tang et al. imply a strong decrease of the bulk modulus with the tube radii in this region. This feature contrasts with the calculated behavior of $\mathrm{Lu},{ }^{21}$ which shows the bulk modulus to be a monotonically increasing function of radius. In addition, the computed bulk modulus for $(11,11)$ or equivalent tubes is $25 \mathrm{GPa}$, which is considerably different from the observed results. These discrepancies highlight the inadequacies of the computational procedure employed by this author. Also, Fig. 3 indicates that the SWNTs behave more like coupled deformable tubes rather than the rigid tubes interacting through van der Waals forces for which the calculated bulk modulus is much higher. ${ }^{20}$ It is interesting to contrast this with the estimated bulk modulus of about $1 \mathrm{GPa}$ in the piston cylinder measurements. One may speculate that if this small value of bulk modulus is not due to any remnant porosity in the sample, then the nature of SWNTs deformation under non-hydrostatic and hydrostatic pressures is very different. In addition, recent Raman scattering studies under nonhydrostatic pressures ${ }^{22}$ show that the pressure derivative of the tangential mode frequency is almost twice as compared to the hydrostatic case. This implies that even for the intertubular compression, the mechanism may be consider- 
ably different from what is observed under hydrostatic conditions.

Figure 2 shows another remarkable feature, i.e., $d_{100}$ of the SWNT bundles relaxes to a higher value at $\sim 9 \mathrm{GPa}$, just before the material undergoes the phase transformation. Reliability of our data can be inferred from the agreement of the observed pressure variation of the $d(002)$ and $d$ (101) diffraction lines of graphite with those published earlier. ${ }^{23}$ The graphite diffraction lines do not show any relaxation effect at $\sim 9 \mathrm{GPa}$, thereby establishing that the SWNTs undergo some structural change around this pressure, probably involving the morphology of the tubes. This is required due to the fact that total compressive strain cannot decrease under the increasing pressure. This observation of relaxation of strain on SWNTs also helps us to understand the Raman results of Ref. 9, where a discontinuous reduction was observed in the tangential mode frequency at $\sim 10 \mathrm{GPa}^{9}{ }^{9} \mathrm{We}$ attribute this to the formation of kinks and fins as described in the MD simulations of Ijima et $_{\text {al. }}{ }^{2}$ and Yakobson, Brabec, and Bernholc. ${ }^{3}$ It should be noted that our data show that the deformation takes place at $\sim \Delta a / a_{0}=6 \%$, which incidentally, is very close to the estimated value of the strain at which the morphological distortions were predicted to start under the axial compression of the isolated tubes in MD simulations. ${ }^{3}$ However, at $\sim 10 \mathrm{GPa}$, the strain along the tube axis is estimated to be $\sim 1 \%$. Therefore, it is unlikely that the present deformations are initiated entirely due to the compression along the tube axis. It may be that the simultaneous presence of basal compressive strain helps trigger the mechanical deformations at a much smaller value of compression along the tube axis. This new feature should spur more theoretical activity. Also, earlier theoretical work has shown that the formation of localized deformation structures release strain on the rest of the tube. ${ }^{2,3}$ However, it may be noted that our experimental results indicate that this relaxation of strain just precedes the loss of translational coherence. This leads us to think that despite the release in the strain, there must be a large enough number of deformed structures in each tube so that a little more compression is adequate to destabilize its two-dimensional lattice structure. This behavior is very different from what has been seen under the nonhydrostatic stresses, where deformation seems to increase monotonically. ${ }^{11}$ In contrast, under the hydrostatic pressures, SWNTs seem to resist morphological deformations up to a critical value of the strain. However, beyond this critical value, the strain releases, probably heterogeneously, and results in a precipitous decline of any translational order at slightly higher compression. This behavior has not been predicted yet in any of the theoretical work and, thus, should prompt more realistic computations. Here it may be noted that the theoretical calculation by Yakobson, Brabec, and Bernholc ${ }^{3}$ had predicted an inward buckling of the tubes at $\sim 1 \mathrm{GPa}$ under the hydrostatic pressures. Our experimental results do not support the existence of such a buckling at such low pressures.
One must note that, in principle, pressure transmitting fluid would interact with SWNTs and, hence, may influence their high-pressure behavior. Presently, not much is known to help address this issue in a detailed manner. In the context of present experiments, one may speculate that pressure transmitting fluid might enter the inter-tubular region and its chemical interaction may bring about morphological changes in the tubes at $\sim 10 \mathrm{GPa}$ at which alcohol mixture solidifies. ${ }^{24}$ However, recent experimental studies indicate that just the solidification of the pressure transmitter may not be the primary driving force for the structural changes observed in SWNTs. ${ }^{25}$ In addition to this, it should also be noted that the observed shifts of the radial modes with pressure strongly disagree with the molecular-dynamics simulations that incorporate the penetration of pressure transmitting fluid between the tubes. ${ }^{6}$

The presence of strong graphite diffraction lines at ambient conditions does not permit the detection of any partial irreversible graphitization of the SWNTs, as suggested in earlier static pressure, ${ }^{10,16}$ as well as shock studies. ${ }^{26}$ The observation of two strong diffraction peaks at $d_{\mathrm{hkl}} \sim 2.5 \AA$, is an interesting feature, not noted earlier. Though its position coincides with one of the diffraction line, (001), of the SWNTs, this line is expected to be much weaker than the first diffraction peak of the sample. Moreover, these diffraction lines continue to be present up to $13 \mathrm{GPa}$, without much change in the diffracted intensity. Further work is necessary to identify the source of this diffraction feature.

To conclude, our high-pressure x-ray diffraction investigations demonstrate that the SWNT bundles lose triangular lattice at $\sim 10 \mathrm{GPa}$. These results supercede the recent observations of loss of the two dimensional lattice at $\sim 1.5 \mathrm{GPa}$ and reversibility of the structural changes up to $\sim 4 \mathrm{GPa}$. We find a reappearance of the lattice on unloading the pressure from $\sim 13 \mathrm{GPa}$. We also note that the volume compression under quasihydrostatic pressures is much less than that under nonhydrostatic stresses. The study underscores the remarkable resilience of nanotube bundles. However, despite the fact that underlying deformations may be quite different, the loss of translational order in SWNTs is similar to that of $\mathrm{C}_{70}$, which shows reversible amorphization and is unlike that of $\mathrm{C}_{60}$, which amorphizes irreversibly. We hope that our work will stimulate theoretical studies to understand the remarkable mechanical properties of carbon nanotube bundles.

\section{ACKNOWLEDGMENTS}

X-ray diffraction experiments were performed at SPring-8 with the approval of Japan Synchrotron Radiation Research Institute (JASRI) Proposal No. 1999B0093-ND-np. We thank the Department of Science and Technology for financial assistance. The authors are grateful to Dr. T. Watanuki for his help during the experiments. 
${ }^{1}$ M. S. Dresselhaus, G. F. Dresselhaus, and P. C. Eklund, Science of Fullerenes and Carbon Nanotubes (Academic Press, New York, 1996), Chap. 19.

${ }^{2}$ S. Ijima, C. Brabec, A. Maiti, and J. Bernholc, J. Chem. Phys. 104, 2089 (1996).

${ }^{3}$ B. I. Yakobson, C. J. Brabec, and J. Bernholc, Phys. Rev. Lett. 76, 2511 (1996).

${ }^{4}$ J. Tersoff and R. S. Ruoff, Phys. Rev. Lett. 73, 676 (1994).

${ }^{5}$ A. Thess, R. Lee, P. Nikolaev, H. Dai, P. Petit, J. Robert, C. Xu, Y. H. Lee, S. G. Kim, A. G. Rinzler, D. T. Colbert, G. E. Scuseria, D. Tomanek, J. E. Fischer, and R. E. Smalley, Science 273, 483 (1996).

${ }^{6}$ U. D. Venkateswaran, A. M. Rao, E. Richter, M. Menon, A. Richter, R. E. Smalley, and P. C. Eklund, Phys. Rev. B 59, 10928 (1999).

${ }^{7}$ M. J. Peters, L. E. McNeil, J. P. Lu, and D. Kahn, Phys. Rev. B 61, 5989 (2000).

${ }^{8}$ A. K. Sood, Pallavi V. Teredesai, D. V. S. Muthu, R. Sen, A. Govindaraj, and C. N. R. Rao, Phys. Status Solidi B 215, 393 (1999).

${ }^{9}$ P. V. Teredesai, A. K. Sood, D. V. S. Muthu, R. Sen, A. Govindraj, and C. N. R. Rao, Chem. Phys. Lett. 319, 296 (2000).

${ }^{10}$ J. Tang, L. C. Qin, T. Sasaki, M. Yudasaka, A. Matsushita, and S. Lijima, Phys. Rev. Lett. 85, 1887 (2000).

${ }^{11}$ S. A. Chesnokov, V. A. Nalimova, A. G. Rinzler, R. E. Smalley, and J. E. Fischer, Phys. Rev. Lett. 82, 343 (1999).

${ }^{12}$ D. W. Snoke, Y. S. Raptis, and K. Syassen, Phys. Rev. B 45, 14419 (1992).

${ }^{13}$ A. K. Sood, N. Chandrabhas, D. V. S. Muthu, Y. Hariharan, A. Bharathi, and C. S. Sundar, Philos. Mag. B 70, 347 (1994).

${ }^{14}$ N. Chandrabhas, A. K. Sood, D. V. S. Muthu, C. S. Sundar, A. Bharathi, Y. Hariharan, and C. N. R. Rao, Phys. Rev. Lett. 73, 3411 (1994).

${ }^{15}$ M. Eswaramoorthy, R. Sen, and C. N. R. Rao, Chem. Phys. Lett. 304, 207 (1999).
${ }^{16}$ V. L. Kuznetsov and V. I. Zaikoviskii (unpublished), J. E. Fischer (unpublished) and cited in Ref. 10.

${ }^{17}$ For SWNTs the tube diameter $d_{t}$ is related to integers $(n, m)$ as $d_{t}=\mathrm{C}_{h} / \pi=\sqrt{ } 3 a_{\mathrm{C}-\mathrm{C}}\left(m^{2}+n^{2}+m n\right)^{1 / 2} / \pi$, where $a_{\mathrm{C}-\mathrm{C}}$ is the nearest-neighbor $\mathrm{C}-\mathrm{C}$ distance $(=1.42 \AA)$ and $\mathrm{C}_{h}$ $=$ is the length of the chiral vector $\mathbf{C}_{\mathbf{h}}=n \mathbf{a}_{\mathbf{1}}+m \mathbf{a}_{\mathbf{2}} ; \mathbf{a}_{\mathbf{1}}, \mathbf{a}_{\mathbf{2}}$ being the primitive vectors of two-dimensional graphene sheet. For Ref. 10 , the tubes diameter is determined to be $14.08 \AA$, therefore, these tubes will correspond to $(10,10)$ armchair or $(18,0)$ zigzag and equivalent tubes.

${ }^{18}$ M. Hanfland, H. Beister, and K. Syassen, Phys. Rev. B 39, 12598 (1989).

${ }^{19}$ F. D. Murnaghan, Proc. Natl. Acad. Sci. U.S.A. 30, 244 (1944).

${ }^{20}$ V. N. Popov, V. E. Van Doren, and M. Balakanski, Solid State Commun. 114, 395 (2000).

${ }^{21}$ J. P. Lu, Phys. Rev. Lett. 79, 1297 (1997).

${ }^{22}$ P. V. Teredesai, A. K. Sood, S. M. Sharma, S. Karmakar, S. K. Sikka, A. Govindarajan, and C. N. R. Rao, Phys. Status Solidi B 223, 479 (2001).

${ }^{23}$ T. Yagi, W. Utsumi, M. Yamakata, T. Kikegawa, and O. Shimomura, Phys. Rev. B 46, 6031 (1992).

${ }^{24}$ Our recorded ruby R-lines spectra did not show any discernible line broadening up to $\sim 11 \mathrm{GPa}$, implying that the nonhydrostatic stresses are small. This is consistent with the fact that though 4:1 methanol-ethanol mixtures solidifies at $\sim 10.4 \mathrm{GPa}$, the resulting solid is a soft one to much higher pressures [Jayaraman, Rev. Mod. Phys. 55, 65 (1983)].

${ }^{25} \mathrm{We}$ rule out this possibility in light of very recent high-pressure Raman experiments (up to $6 \mathrm{GPa}$ ) carried out using water as a pressure-transmitting medium. These measurements do not show any phase transformations up to $6 \mathrm{GPa}$, even though water is known to solidify at $\sim 1 \mathrm{GPa}$ [Pallavi V. Teredèsai and A. K. Sood (unpublished)].

${ }^{26}$ Y. Q. Zhu, T. Sekine, T. Kobayashi, E. Takazawa, M. Terrones, and H. Terrones, Chem. Phys. Lett. 287, 689 (1998). 